\title{
CONTROLLABLE WAVELENGTH CHANNELS FOR MULTIWAVELENGTH BRILLOUIN BISMUTH/ERBIUM BAS-ED FIBER LASER
}

\author{
H. Ahmad, M. Z. Zulkifli, S. F. Norizan, and A. A. Latif \\ Photonics Laboratory \\ Department of Physics \\ University of Malaya \\ Kuala Lumpur 50603, Malaysia
}

\section{S. W. Harun}

Department of Electrical Engineering

Faculty of Engineering

University of Malaya

Kuala Lumpur 50603, Malaysia

\begin{abstract}
We propose and demonstrate a Multiwavelength Brillouin Bismuth/Erbium Fibre Laser (MBBEFL) with the ability to control the number of wavelength channels generated. A multi-wavelength comb output is obtained using a $7.7 \mathrm{~km}$ DCF as a non linear gain medium to generate Stokes wavelengths through the process of Stimulated Brillouin Scattering (SBS). The DCF is pumped by a Raman pump source at $400 \mathrm{~mW}$ to lower the dispersion loss and promote the generation of the Stokes wavelengths. A $49 \mathrm{~cm}$ long Bismuth based Erbium Doped Fibre (Bi-EDF) is used as an optical amplifier to provide the necessary gain for the Brillouin Pump and also act as the gain medium for the generated Stokes signal.
\end{abstract}

\section{INTRODUCTION}

Multi-wavelength sources are critical components for Dense Wavelength Division Multiplexing (DWDM) systems due to their ability to generate many closely-spaced laser wavelengths while at the same time maintaining a simple and inexpensive design, thus ensuring good costper-wavelength. Typically most multi-wavelength sources are based

Corresponding author: H. Ahmad (harith@um.edu.my). 
upon the Erbium Doped Fibres (EDFs) [1]. This is largely due to the fact that EDFs are well understood in terms of their properties and characteristics; in a way providing a clear understanding in the process of generating multi-wavelength outputs from this fibre.

Although the EDF is a good candidate as a linear gain medium for multiwavelength sources, there are however certain drawbacks of using the EDF as the gain medium. The most notable drawback of using the EDF as the gain medium is that multiwavelength lasing at room temperature will be inhibited due to the homogenous broadening mechanism of the Erbium ions at room temperatures, thus putting a limit on the number of oscillating modes. Furthermore, the homogenous broadening mechanism also leads to mode competition and subsequently fluctuating and unstable laser powers [1]. Overcoming this limitation required complex and expensive methods such as cooling the EDF with liquid nitrogen [2] or using specially designed twin-core EDFs [3], which is not practical in real world applications. As a result, other means of overcoming the limitations posed by the homogenous broadening mechanism in the EDF had to be found. This includes the use of Photonic Crystal Fibers (PCFs) and Fiber Bragg Gratings (FBGs) in conjunction with the EDF based multi-wavelength source to generate stable multiwavelength outputs $[4,5]$, as well as utilizing Four-Wave Mixing (FWM) in a non-linear Bismuth-oxide fiber [6] and highly bire-fringent FBGs [7] to overcome the issue of fluctuating powers in the EDF based multiwavelength source. However, these methods require complex setups and are thus not easily applicable for practical applications.

Instead, the multi-wavelength source can be developed without the use of the EDF or other similar materials with homogenous broadening characteristics. A practical approach would be the use of a gain medium with an inhomogeneous broadening mechanism such as the Semiconductor Optical Amplifier (SOA) [8,9]. SOA based multiwavelength sources are not susceptible to limited oscillating modes and mode competition, thus allowing for the generation of multiple stable lasing wavelengths at room temperature. Alternatively, the multi-wavelength source can also be developed by taking advantage of non-linear optical phenomenon such as Stimulated Brillouin Scattering (SBS) [10]. Multi-wavelength Brillouin Fiber Lasers (MBFLs) are based on SBS and generate a closely spaced multiwavelength comb with channel spacings that are determined by the Brillouin frequency shift of $9-12 \mathrm{GHz}$ in the $1550 \mathrm{~nm}$ region, making them attractive for DWDM applications. This generation of Brillouin Stokes are normally performed using a long length of single-mode fibre (usually a length of $25 \mathrm{~km}$ ) which may not be practical in translating 
conventional MBFLs into commercial units. Another shortcoming of this system is that its inability to control the number of wavelengths generated.

In this paper we propose a Multi-wavelength Brillouin Bismuth/Erbium Fiber Laser (MBBEFL) with the ability to control the number of wavelengths generated. The system uses a short length Bismuth-based Erbium Doped Fiber (Bi-EDF) as a linear gain medium and a Dispersion Compensating Fiber (DCF) as a non-linear gain medium. A Raman Pump acting as a pump source for the non-linear gain mechanism and as a stabiliser is also included to enhance the Brillouin channels and stabilize the wavelength channels. A broadband Fiber Bragg Grating (FBG) is employed as a reflector to provide the necessary feedback and to limit the wavelengths generated into the usable region normally used in optical telecommunications. The proposed MBBEFL has the advantage of having a short length non-linear gain medium as well as being able to control the number of Stokes wavelengths generated in the output comb.

\section{EXPERIMENTAL SETUP}

The experimental setup of the proposed MBBEFL is shown in Figure 1 below. The experimental setup consists of three main parts, namely the Bi-EDF based linear amplifier, a non-linear gain medium using a $7.7 \mathrm{~km}$ long Dispersion Compensating Fibre (DCF) and the ring laser cavity, which provides the travelling wave oscillation in the resonator. A broadband FBG is used to provide the necessary reflector for the Stokes signal as discussed in the later part of this paper. In addition, the FBG also provides the added function as a noise filter.

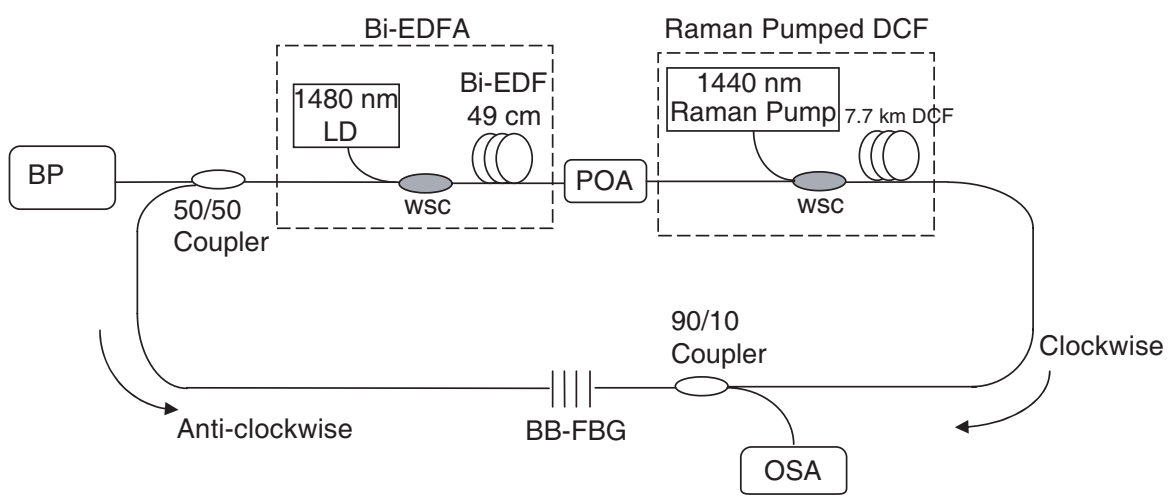

Figure 1. Experimental setup of the proposed MBBEFL. 
The Bi-EDF linear amplifier consists of a $49 \mathrm{~cm}$ Bi-EDF with an erbium ion concentration of $3250 \mathrm{ppm}$. It is pumped by a $1480 \mathrm{~nm}$ laser diode at a power of $160 \mathrm{~mW}$. The pump signal is joined to the ring cavity through a 1480/1550 nm Wavelength Selective Coupler (WSC). The Bi-EDF has a dual role in the proposed setup; it acts as a booster amplifier for the Brillouin Pump (BP) signal generated by the Tunable Laser Source (TLS) and also acts as an in-line amplifier for the subsequently generated Stokes wavelengths. The output of the Bi-EDF is connected to the input of the Programmable Optical Attenuator (POA) in Figure 1. The second part of the proposed setup is the DCF based non-linear gain medium, which consists of a $7.7 \mathrm{~km}$ DCF pumped by a $1440 \mathrm{~nm}$ Raman Pump at $400 \mathrm{~mW}$. Another $1480 / 1550 \mathrm{~nm}$ WSC is employed, with the $1480 \mathrm{~nm}$ and $1550 \mathrm{~nm}$ legs connected to the Raman Pump and POA output respectively, while the common leg is fusion spliced to the DCF. Whilst the DCF acts as a non-linear gain medium, the Raman Pump is used to pump the $\mathrm{DCF}$ and thus reduce the cavity loss, in turn helping to promote the generation of the Stokes wavelengths. Figure 2 shows the On-Off gain characteristics of the DCF which arises from the use of the $1440 \mathrm{~nm}$ Raman Pump, and as shown in the figure the DCF exhibits a very low loss, making it suitable for the generation of the Stokes wavelengths based on SBS. The region at which the On-Off gain is the highest for the DCF is between approximately $1535 \mathrm{~nm}$ and $1545 \mathrm{~nm}$, and thus this is the region at which the BP signal must be generated in order to obtain the most number of high powered Stokes wavelengths.

The third part of the MBBEFL is the ring cavity itself which contains these two important components. The ring cavity is connected

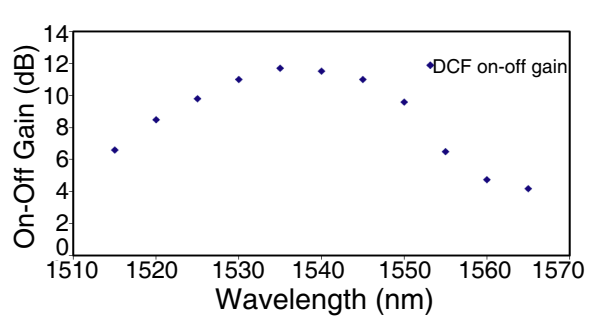

Figure 2. On-off gain profile for $7.7 \mathrm{~km}$ DCF with $400 \mathrm{~mW}$ pumping from the $1440 \mathrm{~nm}$ Raman Pump (The DCF gain is called the on-off gain in accordance to the Raman Pump on-off gain).

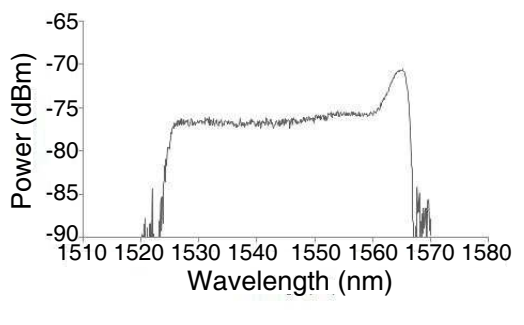

Figure 3. Reflection spectrum of the broadband-FBG. 
to a broadband FBG with a reflectivity of approximately $90 \%$ as well as a $40 \mathrm{~nm}$ bandwidth centred at $1545 \mathrm{~nm}$. In the propose setup, the broadband FBG serves both as an in-line fibre reflector as well as a noise filter. Figure 3 shows the transmission spectrum of the broadband FBG, and it can be seen in the figure that the broadband FBG has a low loss transmission spectrum from $1525 \mathrm{~nm}$ to $1565 \mathrm{~nm}$, giving it the a bandwidth of approximately $40 \mathrm{~nm}$. A 50/50 coupler and a 90/10 tap coupler completes the proposed setup, with the 50/50 coupler acting as an input for the BP signal and the 90/10 tap coupler extracting a portion of the generated signal for analysis by an Optical Spectrum Analyser (OSA) with a resolution of $0.01 \mathrm{~nm}$.

The operation of the proposed MBBEFL is as follows; the BP signal generated by the TLS enters the ring cavity via the 50/50 coupler, and travels in a clockwise direction through the Bi-EDF optical amplifier. In the Bi-EDF based amplifier, the power of the BP signal is increased, and it now travels onward to the DCF. The high power BP signal will now encounter the DCF where it will interact with the non-linear gain medium and form the 1st Brillouin Stokes. The BP signal continues travelling in a clockwise manner towards the broadband FBG, where upon reaching the broadband FBG it will be reflected back in an anti-clockwise direction, through the DCF and BiEDF. It is amplified again by the Bi-EDF and continues travelling in an anti-clockwise direction until it reaches the broadband FBG again, where it will now be reflected back in a clockwise direction. In this manner, the BP signal will oscillate many times in the ring cavity until it drops below its threshold level and stops lasing. As for the 1st Stokes wavelength, upon generation at the DCF it will travel in an anti-clockwise direction towards the Bi-EDF, where it is amplified before continuing onwards to the broadband FBG. At the broadband FBG, the 1st Stokes is reflected and now travels in a clockwise direction through the Bi-EDF to the DCF, where it will now generate the 2nd Stokes wavelength at an anti-clockwise direction. The 1st Stokes continues in the clockwise direction until it encounters the broadband FBG again and is now reflected back in an anti-clockwise direction in the ring cavity. In the same manner as the BP, the 1st Stokes will continue to oscillate in the cavity until it drops below its threshold power and stops lasing. The same process repeats itself in the proposed MBBEFL multiple times, generating a multi-wavelength output comb. The 90/10 tap coupler extracts a portion of the output comb for analysis by the OSA. 


\section{RESULT AND DISCUSSIONS}

As well as acting as an in-line reflector, the broadband FBG also serves another important function; it is necessary for the generation of a high peak power and stable multi-wavelength output comb. Figure 4 shows the spectra profiles produced with and without the inclusion of broadband FBG.

Without the presence of the FBG, the MBBEFL behaves much differently than that of the proposed design. This is because by removing the FBG, the $\mathrm{BP}$ and Stokes will no longer reflect back, instead travelling only in their original direction. This creates a series of alternating clockwise and anti-clockwise Stokes wavelengths (with the odd Stokes wavelengths travelling anti-clockwise and the even Stokes wavelengths travelling clockwise through the ring cavity). As a result, uneven peak powers as observed in Figure 4(b), with fluctuations of almost $5 \mathrm{~dB}$ between the higher and lower powered Stokes wavelengths. However with the broadband FBG, the BP and Stokes wavelengths travel through the MBBEFL continuously and in both directions from being reflected by the broadband FBG, thus giving a relatively more stable output comb, and the overall output comb has a higher power than that of the setup without the broadband FBG.

The effect of the Raman Pump on the proposed MBBEFL is also significant and is shown in Figure 5. As explained in the experimental setup, the Raman Pump is used to reduce the loss dissipation in the DCF, thus promoting the generation of the Stokes wavelengths.

Figure 5(a) shows the emitted signal from the DCF in the ring cavity without the Raman Pump. The loss in the DCF can be high and it will inhibit the generation of multiple Stokes lines. In this figure only the Brillouin pump signal is present and no Stokes lines are observed. However, with the addition of the Raman Pump

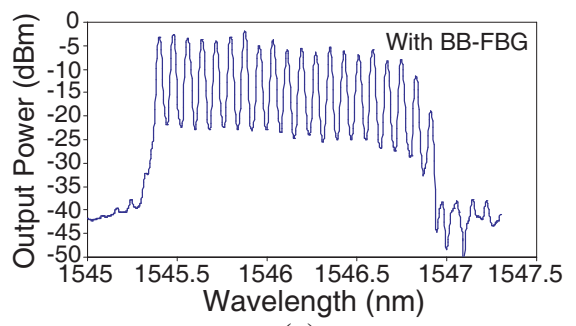

(a)

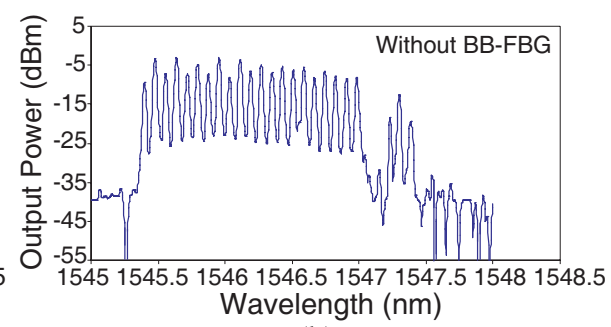

(b)

Figure 4. Comparison of obtained multi-wavelength spectra (a) with and (b) without the broadband FBG in the proposed MBBEFL setup. 
approximately 20 Stokes wavelength channels are generated, as shown in Figure 5(b). Further Stokes wavelengths are also generated, but due to the incorporation of the broadband FBG these wavelengths thus fall outside the reflection spectrum range and are filtered out. The generation of the 20 Stokes wavelengths is further supported by Bi-EDF acting as an amplifier, thus providing gain to the Stokes wavelengths. Figure 6 shows the output of the MBBEFL under different POA attenuation settings.

As can be seen in Figure 6, the proposed MBBEFL has the ability to generate a set number of wavelengths as determined by the attenuation of the POA. The POA controls the power of the BP and the subsequent Stokes to the DCF, thus controlling the number of further Stokes wavelengths that can be generated. It can be seen clearly that as the POA attenuation decreases (thus increasing the power of the $\mathrm{BP}$ and subsequent Stokes wavelengths interacting with the DCF), more and more Stokes wavelengths are generated. In Figure 6(a), the POA attenuation is first set to $10 \mathrm{~dB}$, and it can be seen that only one wavelength is observed in the output, with this wavelength being the BP. However, as the attenuation is reduced to $7 \mathrm{~dB}$, two wavelengths are now obtained as shown in Figure 6(b). The two peaks are observed to have a channel spacing of approximately $0.08 \mathrm{~nm}$, and due to the limitation of the OSA resolution the output spectrum is seen to combine in the middle. Reducing the attenuation further to $5.8 \mathrm{~dB}$ now gives three wavelengths in the output comb as seen in Figure 6(c). Therefore, it can be seen that reducing the attenuation does allow for more wavelengths to be generated as predicted. In the same manner, as the attenuation is brought down to $0 \mathrm{~dB}$, the most number of Stokes wavelengths is 20 wavelengths as shown in Figure 6(f), which is the maximum number of wavelengths that can be obtained in this setup. The channel spacing for all obtained results is approximately $0.08 \mathrm{~nm}$ which is primarily due to the SBS effect. The average power of the

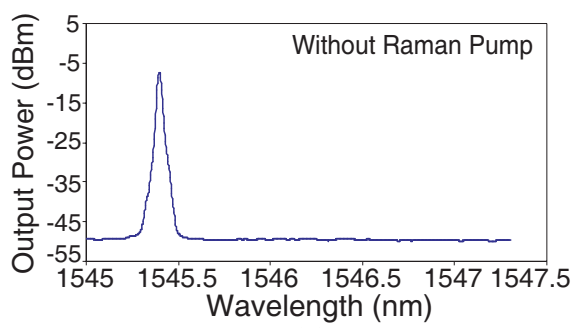

(a)

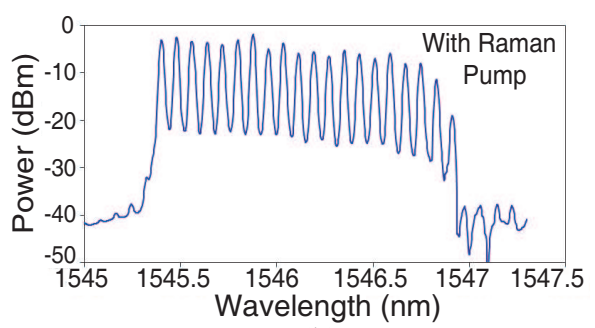

(b)

Figure 5. Multi-wavelength output spectra obtained (a) without and (b) with the Raman pump. 
Ahmad et al.

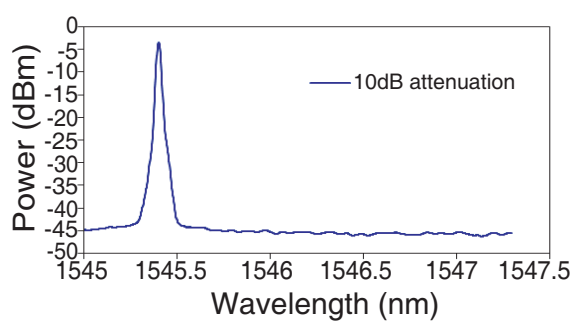

(a)

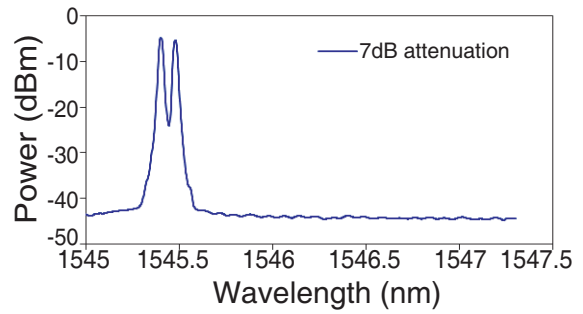

(b)

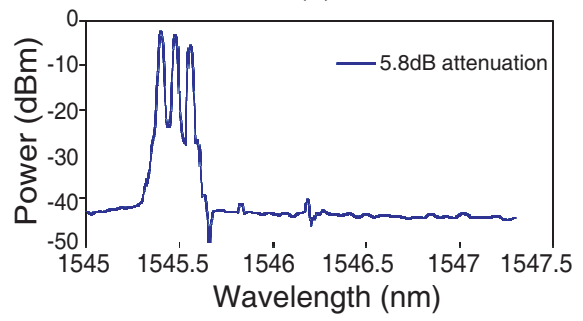

(c)

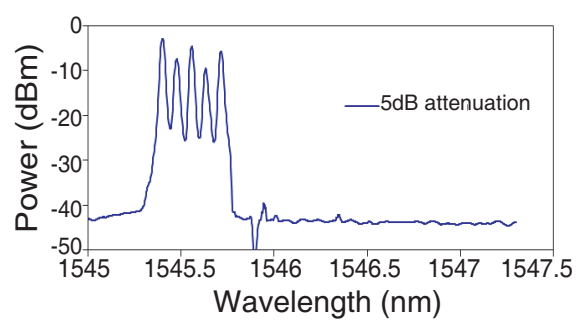

(d)

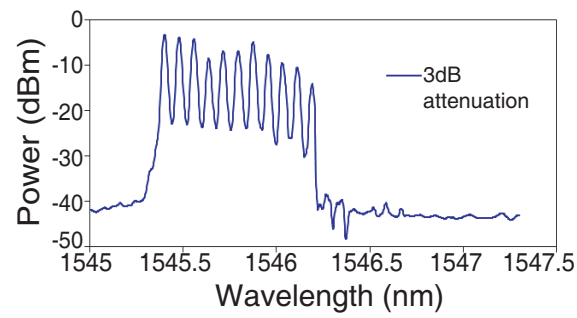

(e)

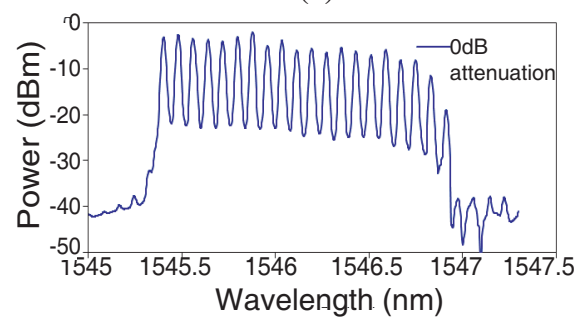

(f)

Figure 6. Multi-wavelength output comb spectra for different POA attenuation values of (a) $10 \mathrm{~dB}$, (b) $7 \mathrm{~dB}$, (c) $5.8 \mathrm{~dB}$, (d) $5 \mathrm{~dB}$, (e) $3 \mathrm{~dB}$, and (f) $0 \mathrm{~dB}$.

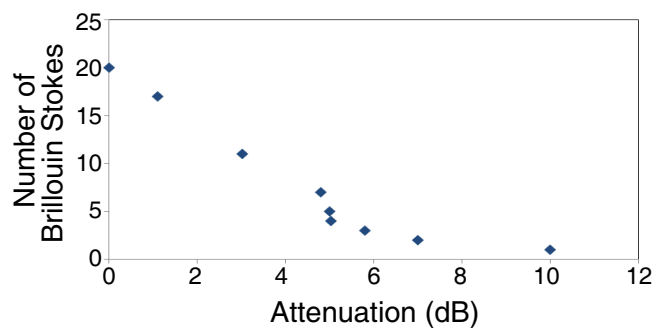

Figure 7. Number of wavelengths obtained for different POA attenuation values $(\mathrm{dB})$. 
lasing wavelengths lies between -4 and $-10 \mathrm{dBm}$. Figure 7 shows the number of wavelengths that can be obtained for POA attenuation values of between 10 to $0 \mathrm{~dB}$.

Figure 7 shows that with the increase of the POA attenuation, the number of wavelengths that can be obtained from the MBBEFL multi-wavelength output comb reduces. It can be seen that initially the reduction of the number of wavelengths to the increase of the attenuation is almost linear. However, at an attenuation of approximately $5 \mathrm{~dB}$, the number of generated wavelengths drops significantly even though the increase of the attenuation in minimal. This is because at this point, the combined DCF and system loss is high enough to suppress the generation of further Stokes, thus from this point onwards only the very high powered BP, 1st and 2nd Stokes can continue to oscillate in the system.

The proposed MBBEFL has the advantage of being compact due to the use of the short Bi-EDF and short DCF as compared to conventional SBS based multi-wavelength sources. Furthermore, the ability to control the number of generated wavelengths is a merit in DWDM systems, allowing the network to adapt to current and changing demands.

\section{CONCLUSION}

In this paper a MBBEFL with the ability to control the number of wavelength channels is demonstrated. A multi-wavelength comb output is generated from Stokes wavelengths through the use of a $7.7 \mathrm{~km}$ DCF which acts as the non linear medium. Optical amplification is provided for by a $49 \mathrm{~cm}$ Bi-EDF based amplifier. A $400 \mathrm{~mW}$ Raman pump is also added as a pump source for the DCF to overcome the attenuation in the fibre and promote the generation of the Stokes wavelengths. A range of 1 to 20 Stokes is generated for an attenuation of 10 to $0 \mathrm{~dB}$ at a wavelength region of 1545.3 to $1546.9 \mathrm{~nm}$, with a channel spacing of $0.08 \mathrm{~nm}$ and an average peak power of between -5 and $-10 \mathrm{dBm}$. The addition of a broadband FBG provides a more stable multi-wavelength comb with minimal power fluctuations.

\section{REFERENCES}

1. Mears, R. J., L. Reekie, I. M. Jauncey, and D. N. Payne, "Lownoise erbium-doped fibre amplifier at 1.54 pm," Electron. Lett., Vol. 23, 1026-1028, 1987. 
2. Chow, J., G. Town, B. Eggleton, M. Ibsen, K. Sugden, and I. Bennion, "Multiwavelength generation in an erbium-doped fiber laser using in-fiber comb filters," IEEE Photon. Technol. Lett., Vol. 8, 60-62, 1996.

3. Dong, X. P., S. P. Li, K. S. Chiang, M. N. Ng, and B. C. B. Chu, "Multiwavelength erbium-doped fibre laser based on a highbirefringence fibre loop mirror," Electron. Lett., Vol. 36, 16091610, 2000.

4. Liu, X., X. Yang, F. Lu, J. Ng, X. Zhou, and C. Lu, "Stable and uniform dual wavelength erbium-doped fiber laser based on fiber Bragg gratings and photonic crystal fiber," Opt. Express, Vol. 13, 142-147, 2005.

5. Yang, X., X. Dong, S. Zhang, F. Lu, X. Zhou, and C. Lu, "Multiwavelength erbium-doped fiber laser with 0.8-nm spacing using sampled Bragg grating and photonic crystal fiber," IEEE Photon. Technol. Lett., Vol. 17, 2538-2540, 2005.

6. Fok, M. P. and C. Shu, "Tunable dual-wavelength erbium-doped fiber laser stabilized by four-wave mixing in a $35-\mathrm{cm}$ highly nonlinear bismuth-oxide fiber," Opt. Express, Vol. 15, No. 10, 5925-5930, 2007.

7. Liu, Y., X. Feng, S. Yuan, G. Kai, and X. Dong, "Simultaneous four-wavelength lasing oscillations in an erbium-doped fiber laser with two high birefringence fiber Bragg gratings," Opt. Express, Vol. 12, 2056-2061, 2004.

8. Harun, S. W., M. Z. Zulkifli, and H. Ahmad, "A linear cavity S-band Brillouin/erbium fiber laser," Laser Phys. Lett., Vol. 3, No. 7, 369-371, 2006.

9. Han, Y. G., G. Kim, J. H. Lee, S. H. Kim, and S. B. Lee, "Lasing wavelength and spacing switchable multiwavelength fiber laser from 1510 to $1620 \mathrm{~nm}$," IEEE Photon. Technol. Lett., Vol. 17, 989-991, 2005.

10. Harun, S. W., S. Shahi, and H. Ahmad, "Compact Brillouinerbium fiber laser," Opt. Express, Vol. 34, 46-48, 2009.

11. Shahi, S., S. W. Harun, A. H. Sulaiman, K. Thambiratnam, and H. Ahmad, "Multiwavelength source based on SOA and EDFA in a ring-cavity resonator," Microwave and Optical Tech. Lett., Vol. 51, No. 1, 110-113, 2008.

12. Fok, M. P. and C. Shu, "Tunable dual-wavelength erbium-doped fiber laser stabilized by four-wave mixing in a 35-cm highly nonlinear bismuth-oxide fiber," Opt. Express, Vol. 15, No. 10, 5925-5930, 2006. 